\title{
CARACTERÍSTICAS SOCIODEMOGRÁFICAS E CONDIÇÕES DE SAÚDE DE IDOSAS LONGEVAS INATIVAS FISICAMENTE PARTICIPANTES DE GRUPOS DE CONVIVÊNCIA PARA IDOSOS
}

DOI: 105902/0102830810532

Data de submissão: 28/08/2013 Data de Aceite: 02/04/2014

Rodrigo de Rosso Krug

Universidade Federal de Santa Catarina rodkrug@bol.com.br

Marize Amorim Lopes

Universidade Federal de Santa Catarina marize.amorim@ufsc.br

Giovana Zarpellon Mazo

Universidade Federal de Santa Catarina

giovana.mazo@udesc.br

RESUMO: Objetivou-se identificar as características sociodemográficas e as condições de saúde das idosas longevas inativas fisicamente participantes de grupos de convivência para idosos de diferentes regiões de Florianópolis-SC. Foram estudadas 173 idosas inativas fisicamente, com 80 anos ou mais, frequentadoras dos grupos de convivência de Florianópolis-SC. Aplicou-se entrevista estruturada, contendo dados sociodemográficos, condições de saúde e o Questionário Internacional de Atividade Física adaptado para idosos. A média de idade foi 84,9 94,12 anos, sendo a maioria viúva, com baixa escolaridade, católica, caucasiana, aposentada/pensionista, apresenta alguma doença, usa medicamentos e não praticavam atividade física moderada ou vigorosa, por pelo menos 10 minutos contínuos durante uma semana normal, nos quatro domínios do IPAQ. Espera-se que estes resultados auxiliem no planejamento de estratégias de intervenções que busquem modificações de comportamentos para a adoção de um estilo de vida ativo desta população.

Palavras-chave: Idosas com idade igual ou superior a 80 anos. Características sociodemográficas. Condições de saúde. Nível de atividade física. 


\section{Introdução}

A população com 80 anos ou mais vem aumentando rapidamente no Brasil. Em 2010 foram identificados no Brasil mais de três milhões de pessoas nessa faixa etária, com projeções de aumento para 14 milhões em 2040. Esses são denominados de idosos longevos e apresentam características morfofisiológicas, psicológicas e socioeconômicas diferenciadas, além de um crescente número de morbidades e incapacidades (IBGE, 2010; NOGUEIRA et al., 2010).

Estas características contribuem para que estas pessoas mais velhas tenham um aumento do risco de inatividade física com o passar dos anos (HIRVENSALO; LINTUNEN, 2011; VON BONSDORFF; RANTANEN, 2011; MECHLING; NETZ, 2009). O estudo epidemiológico EpiFloripa Idoso, que avaliou 1705 idosos residentes em Florianópolis-SC, verificou que $42,1 \%$ desses idosos são inativos fisicamente, sendo que se considerarmos somente os idosos longevos $(n=214)$ essa prevalência aumenta para 58,4\% (CORSEUIL et al., 2011).

A inatividade física age como acelerador do declínio humano, ocasionando maior incapacidade funcional, perda de qualidade de vida, aumento do número de doenças, obesidade e mortalidade (MECHLING; NETZ, 2009) entre outras consequências. Nesse contexto, a prática de atividade física regular vem sendo amplamente recomendada por profissionais e organizações de saúde como uma forma de promover a saúde, onde a participação em atividades físicas regulares pode diminuir o aparecimento de doenças crônicas (ACSM, 2009). Nesse sentido, a participação de idosos longevos em programas de atividades físicas pode minimizar os efeitos deletérios do envelhecimento.

Pesquisas nessa temática tornam-se relevantes, devido aos seguintes aspectos: a expectativa de vida do brasileiro vem aumentando consideravelmente, passando de 67 anos, em 1991, para 72,8 anos, em 2010 (IBGE, 2010); os estudos nacionais são mais direcionados aos idosos jovens e ativos fisicamente que apresentam características e necessidades diferenciadas do idoso longevo; maior participação de idosos jovens em programas de atividades físicas regulares (MEURER; BENEDETTI; MAZO, 2011); e que apenas $5 \%$ das pessoas com 60 anos ou mais residentes em Florianópolis-SC participam de programas de atividade física (BENEDETTI; GONÇALVES; MOTA, 2007). 
Assim, surge a necessidade de estudos para o melhor conhecimento das características dos idosos longevos, com o intuito de melhores intervenções, visando a maior participação desses em práticas regulares de atividades físicas. Com isso, objetivou-se neste estudo, identificar as características sociodemográficas e as condições de saúde das idosas longevas inativas fisicamente participantes de grupos de convivência para idosos de diferentes regiões de Florianópolis-SC.

\section{Método}

Tipo De Pesquisa e Aspectos Éticos

Os dados deste estudo descritivo epidemiológico transversal foram coletados nos anos de 2010 e 2011. O mesmo faz parte da pesquisa "Idosos Longevos: estilo de vida e percepção sobre a prática de exercícios físicos e hábitos de lazer" que foi aprovada no Comitê de Ética Envolvendo Seres Humanos da Universidade do Estado de Santa Catarina (UDESC), parecer n 149/2010.

\section{População e Amostra}

A população deste estudo envolveu 493 idosos com 80 anos ou mais de idade, participantes de pelo menos um dos 102 grupos de convivência para idosos, cadastrados na Prefeitura Municipal de Florianópolis-SC.

Grupos de convivência para idosos são baseados em práticas educativas desenvolvidas em grupos, que tem propósitos ou metas comuns. Proporcionando uma educação participativa, na perspectiva de que todos se mostrem ativos no trabalho grupal. Nos grupos de convivência cadastrados no município são desenvolvidas atividades manuais (tricô, crochê, corte e costura), recreativas (bingo, cartas e dominó), educativas (palestras e cursos), viagens turísticas e lanches. Geralmente os grupos se reúnem uma vez por semana (PMF, 2009).

A amostra foi selecionada de forma intencional e voluntária, onde participaram idosos com 80 anos ou mais que estavam presentes no dia das coletas e que aceitaram participar voluntariamente da pesquisa. Foi realizada somente uma visita em cada grupo 
de convivência. Sendo assim, foram avaliados 351 idosos longevos, sendo que destes, 28 eram do sexo masculino e 323 do feminino, onde 173 eram idosas longevas inativas fisicamente (Quadro 1).

Optou-se, neste estudo, pelas mulheres idosas longevas inativas fisicamente $(n=173)$, devido ao maior número delas nos grupos de convivência e ao maior número de mulheres neste estrato etário mais longevo. Este fato de existirem mais mulheres idosas do que homens é denominado de feminização da velhice (NERI, 2007). Estudos com idosos longevos também apontam maior proporção de mulheres neste estrato etário (ZIBETTI et al., 2010; SCHMIDT et al., 2009).

Quadro 1: População e amostra do estudo: Número de grupos de convivência por regiões de Florianópolis-SC, quantidade de idosos longevos cadastrados, entrevistados e inativos fisicamente.

\begin{tabular}{|l|c|c|c|c|c|}
\hline \multirow{2}{*}{ Região } & \multirow{2}{*}{$\begin{array}{c}\mathbf{N}^{0} \text { de } \\
\text { grupos de } \\
\text { convivência }\end{array}$} & \multirow{2}{*}{$\begin{array}{c}\mathbf{N}^{\mathbf{0}} \text { de } \\
\text { longevos } \\
\text { cadastrados }\end{array}$} & \multicolumn{2}{|c|}{$\mathbf{N}^{\mathbf{2}}$ de longevos entrevistados } & \multirow{2}{*}{$\begin{array}{c}\mathbf{N}^{0} \text { de idosas } \\
\text { longevas } \\
\text { inativas } \\
\text { fisicamente }\end{array}$} \\
\cline { 4 - 6 } & & & Homens & Mulheres & 52 \\
\hline Centro & 31 & 168 & 6 & 96 & 12 \\
\hline Leste & 9 & 45 & 5 & 32 & 41 \\
\hline Sul & 18 & 86 & 8 & 60 & 24 \\
\hline Norte & 16 & 75 & 5 & 51 & 44 \\
\hline Continente & 28 & 119 & 4 & 323 & 173 \\
\hline Total & 102 & 493 & 28 & & \\
\hline
\end{tabular}

\section{Instrumentos}

Os instrumentos utilizados na pesquisa foram:

- Ficha diagnóstica com 23 questões abertas e fechadas sobre as características sociodemográficas e condições de saúde.

- Questionário Internacional de Atividade Física (IPAQ), forma longa, semana normal, adaptado para idosos (MAZO; BENEDETTI, 2010), que avalia o nível de atividade física. Esse é um instrumento que permite estimar o dispêndio energético semanal de atividades físicas relacionadas com trabalho, transporte, tarefas domésticas, lazer e na posição sentada, com intensidade moderada ou vigorosa, durante 10 minutos contínuos, numa semana normal (MARSHALL; BAUMAN, 2001; MAZO; BENEDETTI, 2010). 


\section{Coleta de Dados}

Primeiramente, contatou-se com a Prefeitura Municipal de Florianópolis-SC para se ter acesso ao número, ao endereço dos grupos de convivência para idosos cadastrados na mesma, e os dados para contato com os coordenadores dos grupos. A Gerência do Idoso da Prefeitura Municipal de Florianópolis-SC forneceu o banco de dados, onde se identificaram o local de funcionamento e os telefones das coordenadoras dos 102 grupos de convivência.

Posteriormente, foram contatados os coordenadores dos grupos de convivência para pedir permissão para fazer a pesquisa nos mesmos. A etapa seguinte foi identificar quantos idosos longevos existiam cadastrados nesses grupos, para convidá-los a participarem do estudo e esclarecer os objetivos deste.

As idosas longevas foram contatadas no próprio grupo de convivência de que participavam, onde foi realizado o convite para a participação na pesquisa e marcada data e horário para esta. Ao aceitarem participar da pesquisa, foi assinado o Termo de Consentimento Livre e Esclarecido em duas vias, ficando uma na posse do pesquisador e a outra na posse do participante. Após, foi aplicada, em forma de entrevista, a ficha diagnóstica e o IPAQ.

\section{Tratamento dos Dados}

Os dados provenientes da ficha diagnóstica foram analisados a partir da estatística descritiva, por meio de média e frequência simples no programa SPSS 17.0 for Windows.

Para analisar o nível de atividade física dos idosos longevos a partir do IPAQ, foi utilizado o somatório dos tempos (minutos por semana) das atividades físicas moderadas e vigorosas nos seus diferentes domínios (trabalho, transporte, tarefas domésticas e lazer). A definição de inativa fisicamente se deu neste estudo com base nas recomendações de limiares de atividades físicas que resultam em benefícios para a saúde (MARSHALL; BAUMAN, 2001), ou seja, pessoas que praticam menos de 150 minutos por semana de atividades físicas moderadas e/ou vigorosas. 


\section{Resultados}

A média de idade das 173 idosas longevas inativas fisicamente foi de $84,9 \pm 4,12$ anos. Pode-se verificar na tabela 1 que a maioria delas era viúva $(82,1 \%)$, morava acompanhada $(67,6 \%)$, tinha o ensino fundamental incompleto $(60,1 \%)$, católica $(95,4 \%)$, caucasiana $(94,8 \%)$, aposentada/pensionista $(95,9 \%)$, apresentava alguma doença $(90,8 \%)$ e fazia uso de medicamento regularmente (90,2\%). Além disso, 43,9\% recebiam entre um e três salários mínimos mensais e $42,8 \%$ consideravam sua saúde regular (42,8\%). As características sociodemográficas foram semelhantes para as idosas das diferentes regiões de Florianópolis, bem como para as condições de saúde, com exceção para o aspecto do estado de saúde atual que as idosas das regiões norte, centro e continente consideraram sua saúde ótima/boa.

Tabela 1: Características sociodemográficas e as condições de saúde das idosas longevas inativas fisicamente participantes de grupos de convivência nas diferentes regiões (Centro, Sul, Norte, Leste e Continente) de Florianópolis-SC ( $n=173)$. Florianópolis-SC, 2011.

\begin{tabular}{|c|c|c|c|c|c|c|}
\hline Variáveis & $\begin{array}{l}\text { Norte } \\
\mathrm{f}(\%)\end{array}$ & $\begin{array}{l}\text { Leste } \\
\text { f (\%) }\end{array}$ & $\begin{array}{c}\text { Centro } \\
\mathrm{f}(\%)\end{array}$ & $\begin{array}{c}\text { Sul } \\
\mathrm{f}(\%)\end{array}$ & $\begin{array}{l}\text { Cont. } \\
\text { f (\%) }\end{array}$ & $\begin{array}{l}\text { Total } \\
\mathrm{f}(\%)\end{array}$ \\
\hline \multicolumn{7}{|l|}{ Estado Civil } \\
\hline Solteiro & $0(0)$ & $1(8,3)$ & $8(15,4)$ & $1(2,4)$ & $3(6,8)$ & $13(7,5)$ \\
\hline Casado & $0(0)$ & $1(8,3)$ & $3(5,8)$ & $5(12,2)$ & $4(9,1)$ & $13(7,5)$ \\
\hline Separado & $1(4,2)$ & $0(0)$ & $3(5,8)$ & $0(0)$ & $1(2,3)$ & $5(2,9)$ \\
\hline Viúvo & $23(95,8)$ & $10(83,4)$ & $38(73)$ & $35(85,4)$ & $36(81,8)$ & $142(82,1)$ \\
\hline \multicolumn{7}{|l|}{ Com quem mora } \\
\hline Sozinho & $8(33,3)$ & $3(25)$ & $16(30,8)$ & $16(39)$ & $13(29,5)$ & $56(32,4)$ \\
\hline Acompanhado & $16(66,4)$ & $9(75)$ & $36(69,2)$ & $25(61)$ & $31(70,5)$ & $117(67,6)$ \\
\hline \multicolumn{7}{|l|}{ Escolaridade } \\
\hline Analfabeto & $4(16,8)$ & $2(16,7)$ & $8(15,4)$ & $6(14,6)$ & $6(13,6)$ & $26(15,0)$ \\
\hline Fundamental Incompleto & $18(75)$ & $7(58,3)$ & $22(42,3)$ & $29(70,7)$ & $28(63,6)$ & $104(60,1)$ \\
\hline Fundamental Completo & $1(4,1)$ & $3(25)$ & $10(19,2)$ & $4(9,8)$ & $5(11,5)$ & $23(13,3)$ \\
\hline Médio Incompleto & $0(0)$ & 0 & $1(1,9)$ & $0(0)$ & $2(4,5)$ & $3(1,7)$ \\
\hline Médio Completo & $1(4,1)$ & 0 & $8(15,4)$ & $2(4,9)$ & $1(2,3)$ & $12(6,9)$ \\
\hline Ensino Superior & $0(0)$ & 0 & $3(5,8)$ & $0(0)$ & $2(4,5)$ & $5(2,9)$ \\
\hline \multicolumn{7}{|l|}{ Religião } \\
\hline Católico & $24(100)$ & $11(91,7)$ & $49(94,2)$ & $39(95,2)$ & $42(95,5)$ & $165(95,4)$ \\
\hline Evangélico & $0(0)$ & $0(0)$ & $0(0)$ & $0(0)$ & $0(0)$ & $0(0)$ \\
\hline Espirita & $0(0)$ & $1(8,3)$ & $1(1,9)$ & $1(2,4)$ & $2(4,5)$ & $5(2,9)$ \\
\hline Sem religião & $0(0)$ & $0(0)$ & $2(3,8)$ & $0(0)$ & $0(0)$ & $2(1,2)$ \\
\hline Outros & $0(0)$ & $0(0)$ & $0(0)$ & $1(2,4)$ & $0(0)$ & $1(0,6)$ \\
\hline \multicolumn{7}{|l|}{ Etnia } \\
\hline Caucasiano & $23(95,8)$ & $12(100)$ & $50(96,2)$ & $36(87,8)$ & $43(97,7)$ & $164(94,8)$ \\
\hline Afrodescendentes & $1(4,2)$ & $0(0)$ & $2(3,8)$ & $5(12,2)$ & $1(2,3)$ & $9(5,2)$ \\
\hline \multicolumn{7}{|l|}{ Ocupação } \\
\hline Aposentado/Pensionista & $24(100)$ & $11(91,7)$ & $51(98,1)$ & $39(95,2)$ & $42(95,5)$ & $108(95,9)$ \\
\hline Aposentado/Pensionista ativo & $0(0)$ & $1(8,3)$ & $0(0)$ & $1(2,4)$ & $0(0)$ & $2(1,2)$ \\
\hline Não remunerado & $0(0)$ & $0(0)$ & $1(1,9)$ & $1(2,4)$ & $2(4,5)$ & $4(2,3)$ \\
\hline
\end{tabular}




\begin{tabular}{l|c|c|c|c|c|c|} 
Renda & & & \\
< 1 Salário minimo & $2(8,3)$ & $4(33,3)$ & $10(19,2)$ & $9(22)$ & $7(15,9)$ & $32(18,5)$ \\
1 a 3 Salários minimos & $18(75,0)$ & $2(16,7)$ & $18(34,7)$ & $20(48,8)$ & $22(50,0)$ & $80(43,9)$ \\
3 a 4 Salários mínimos & $1(4,2)$ & $3(25)$ & $4(7,7)$ & $3(7,3)$ & $4(9,1)$ & $15(8,7)$ \\
4 a 6 Salários mínimos & $2(8,3)$ & $2(16,7)$ & $5(9,6)$ & $6(14,6)$ & $2(4,5)$ & $17(9,8)$ \\
$\quad$ > 6 Salários minimos & $1(4,2)$ & $1(8,3)$ & $15(28,8)$ & $3(7,3)$ & $9(20,5)$ & $29(16,8)$ \\
\hline Estado de saúde & & & & & & \\
Ótimo/Bom & $11(45,9)$ & $4(33,3)$ & $22(42,3)$ & $12(29,3)$ & $20(45,4)$ & $69(39,9)$ \\
Regular & $10(41,7)$ & $5(41,7)$ & $21(40,4)$ & $22(53,7)$ & $16(36,4)$ & $74(42,8)$ \\
Ruim/Muito Ruim & $3(12,4)$ & $3(25)$ & $9(17,3)$ & $7(17)$ & $8(18,2)$ & $30(17,4)$ \\
\hline Doenças & & & & & & \\
Sim & $21(87,5)$ & $11(91,7)$ & $49(94,2)$ & $36(87,8)$ & $40(90,9)$ & $157(90,8)$ \\
Não & $3(12,5)$ & $1(8,3)$ & $3(5,8)$ & $5(12,2)$ & $4(9,1)$ & $16(9,2)$ \\
\hline Medicamentos & & & & & & \\
Sim & $20(83,3)$ & $12(100)$ & $48(92,3)$ & $35(85,4)$ & $41(93,2)$ & $156(90,2)$ \\
Não & $4(16,7)$ & $0(0)$ & $4(7,7)$ & $6(14,6)$ & $3(6,8)$ & $17(9,8)$ \\
\hline
\end{tabular}

Legenda: $\mathrm{f}=$ Frequência; $\%=$ percentagem; Cont.=Continente .

No presente estudo, as mulheres idosas longevas foram consideradas inativas fisicamente, pois praticavam menos de 150 minutos por semana de atividades físicas moderadas e/ou vigorosas, por meio do IPAQ adaptado para idosos por Mazo e Benedetti (2010). Na soma total dos quatro domínios do IPAQ (trabalho, transporte, atividades domesticas e lazer) das 173 idosas longevas inativas fisicamente, 59\% praticavam de 10 a 149 minutos por semana de atividades físicas moderadas ou vigorosas e $41 \%$ não praticavam atividade física moderada ou vigorosa, por pelo menos 10 minutos contínuos durante uma semana normal.

Ao analisar separadamente pelos domínios do IPAQ, verificou-se, na figura 1, que a maioria das idosas longevas estudadas não praticava atividade física moderada ou vigorosa, por pelo menos 10 minutos contínuos durante uma semana normal, sendo que $64 \%$ no transporte, $77 \%$ nas atividades domésticas e $89 \%$ no lazer. No domínio trabalho todas $(100 \%)$ não praticavam atividade física. 

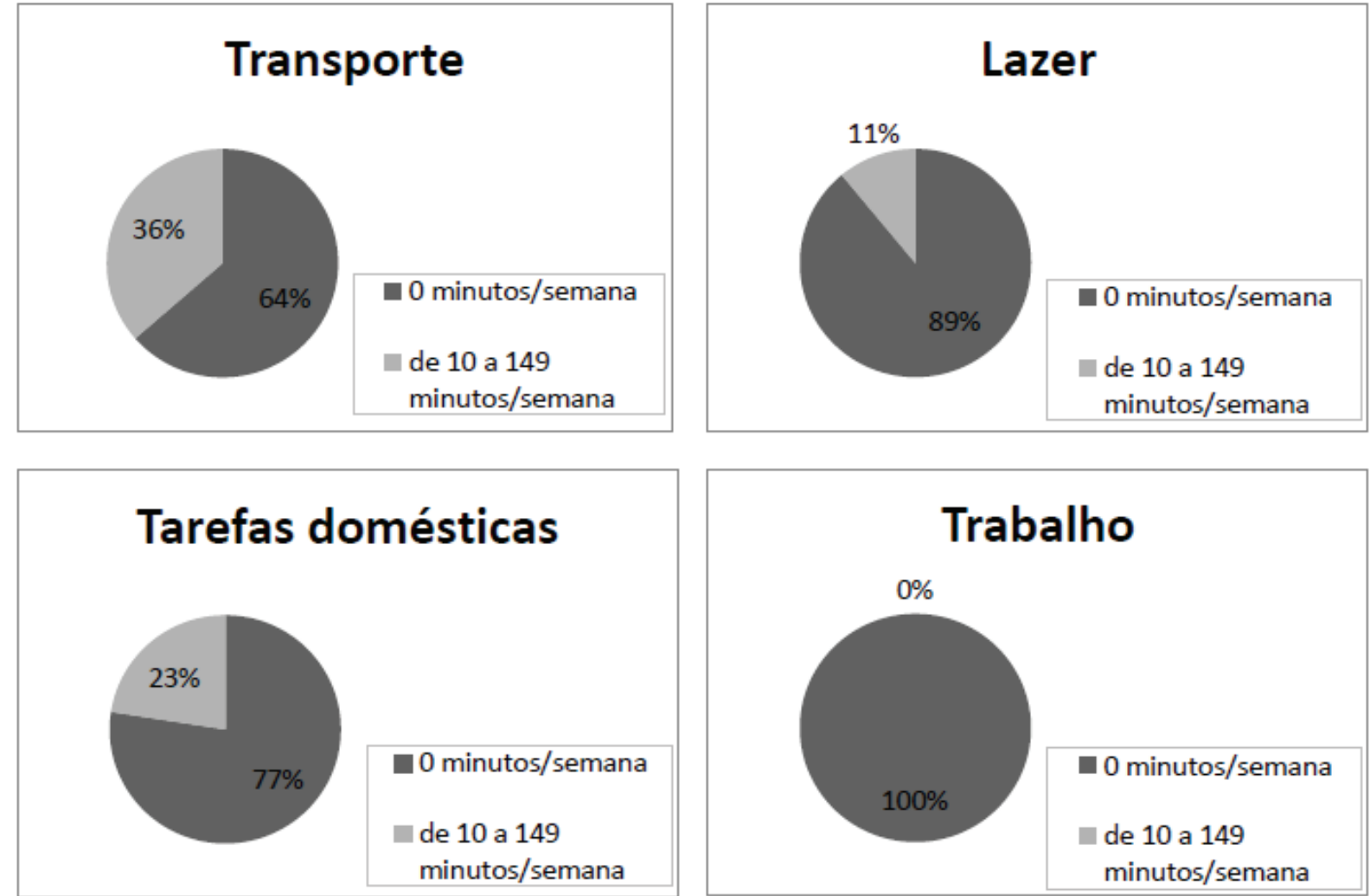

Figura1: Percentual de tempo gasto com atividades físicas semanais (minutos/semana) das idosas longevas inativas fisicamente, de acordo com os domínios do IPAQ.

\section{Discussão}

No presente estudo, para identificar as mulheres idosas longevas inativas fisicamente, utilizou-se o IPAQ adaptado para idosos por Mazo e Benedetti (2010), que por meio da somatória de seus domínios retratou que das 251 idosas longevas entrevistadas, 59\% praticavam de 10 a 149 minutos por semana de atividades físicas moderadas ou vigorosas, sendo consideradas longevas ativas e $41 \%$ foram consideradas longevas inativas fisicamente por não praticarem atividade física moderada ou vigorosa, por pelo menos 10 minutos contínuos durante uma semana normal (Figura 1).

Scarmeas et al. (2001) encontraram relação estatisticamente significativa entre escolaridade e participação de idosos em atividades de lazer. O experimento acompanhou, durante sete anos, 1772 americanos com idade igual ou superior a 65 anos, e demonstrou que, entre os idosos com nível de escolaridade maior, a média de prática de atividades de lazer também foi maior, se comparados aos indivíduos com menor escolaridade. Hallal et al. (2003) explicam que os indicadores sociais, renda e escolaridade, geralmente estão atrelados, onde quem tem maiores níveis de escolaridade geralmente tem maior renda se 
comparados aos de menor escolaridade. Dessa forma, a renda e a escolaridade são vistos como determinantes importantes no estilo de vida das pessoas (HIRVENSALO; LINTUNEN, 2011; HALLAL et al., 2003).

A autopercepção de saúde também foi verificada no presente estudo, onde $42,8 \%$ das idosas longevas relataram que sua saúde era regular. Este resultado também foi evidenciado por Moschny et al. (2011), ao estudarem 1937 idosos alemães com idades entre 72 e 93 anos e verificarem que as pessoas com 80 anos ou mais de idade percebem sua saúde como pior, se comparados a pessoas com menor idade.

A saúde, dentre outros fatores, também influencia na inatividade física diretamente (MOSCHNY et al., 2011; GOBBI et al., 2008). No estudo PENSA (Estudo dos Processos de Envelhecimento Saudável) com 155 idosos (60 anos ou mais) da comunidade de Juiz de Fora/MG, constatou-se, que idosos que avaliaram sua saúde como excelente ou muito boa praticavam atividade física com maior frequência do que aqueles que avaliaram sua saúde como razoável ou péssima (RIBEIRO et al., 2009). Nogueira et al. (2010) verificaram em seu estudo com 129 idosos longevos de São Geraldo/MG, que considerar a própria saúde pior que a de outros idosos foi considerado um fator de risco $(O R=4,40)$ para pior capacidade funcional. Essa perda da capacidade funcional gera uma diminuição nos níveis de atividade física (HIRVENSALO; LINTUNEN, 2011).

As condições de saúde também foram analisadas por meio do uso de medicamentos e presença de doenças, onde foi evidenciado que a maioria das idosas longevas apresenta alguma doença e faz uso de medicamentos regularmente. Alguns pesquisadores (MOREIRA et al., 2007; XAVIER et al., 2006; CRUZ et al., 2004; MARAFON et al., 2003) afirmam que os idosos longevos estão no limite de suas capacidades funcionais, pois esta população geralmente tem uma frequência maior de incapacidade funcional e um número crescente de doenças crônicas. À medida que a pessoa envelhece, maiores são as chances de desenvolver uma doença crônica, onde $80 \%$ dos idosos longevos tem pelo menos uma doença diagnosticada pelo médico e 50,4\% têm duas doenças (IBGE, 2010).

Mesmo em grupos de idosos ativos, a presença de pelo menos uma morbidade é comum. No estudo de Meurer, Benedetti e Mazo (2011) com 111 idosos (60 anos e mais) participantes de um programa de exercícios físicos, 87\% apresentavam alguma doença 
diagnosticada pelo médico. No estudo de Schwanke et al. (2002) com 70 idosos longevos residentes na cidade de Veranópolis/RS evidenciou-se que 95\% possuíam alguma doença relacionada ao envelhecimento.

As doenças mais comuns nessa faixa etária de 80 anos ou mais de idade são: hipertensão (IBGE, 2010; SCHWANKE et al., 2002), acidente vascular cerebral, câncer, doenças cardiovasculares (MOREIRA et al., 2007; XAVIER et al., 2006; CRUZ et al., 2004; MARAFON et al., 2003; SCHWANKE et al., 2002), hipercolesteromia e artrite (IBGE, 2010).

O aumento de doenças leva ao maior uso de medicamentos. Benedetti, Petroski e Gonçalves (2004), traçaram o perfil dos idosos do município de Florianópolis-SC ao avaliarem 875 idosos e observaram que apenas $27 \%$ destes não utilizavam medicamentos. Silva (2009) verificou, em estudo mais recente, que $84,5 \%$ das idosas praticantes de atividades físicas no programa da saúde da família de São Caetano do Sul-SP faziam uso de três ou mais medicamentos por dia.

Ao relacionar o consumo de medicamentos com a prática de atividades físicas, Novais (2009) verificou que, quanto maior o uso de medicamentos diário, maior a chance de a pessoa idosa não praticar atividades físicas no lazer. Entretanto, Nogueira et al. (2010) ao estudarem 129 idosos longevas verificaram que o uso contínuo de cinco ou mais medicamentos $(\mathrm{OR}=2,67)$ pode gerar fatores de riscos para a redução da capacidade funcional.

Em relação aos níveis de atividade física, percebeu-se neste estudo, que nenhuma idosa longeva participante dos grupos de convivência está inserida no mercado de trabalho. Benedetti, Gonçalves e Mota (2007), pesquisaram idosos de Florianópolis/SC com idade igual ou superior a 60 anos, e também constataram que 93\% destes não trabalham. Jardim (2007), relata que o mercado de trabalho, realmente é o primeiro a excluir as pessoas idosas por considerá-las defasadas e com menor capacidade produtiva. Essa exclusão é ainda maior para os idosos longevos. Pela idade avançada das idosas estudadas, também podese atribuir o fato de elas não estarem mais no mercado de trabalho e por não quererem ou poderem mais trabalhar.

Ao estudar 30 ex-ferroviários da Estrada de Ferro Sorocabana, residentes no município de Botucatu/SP, Patrício et al. (2008) concluíram ou que os idosos com histórico de muito 
trabalho, seja em casa ou na lavoura, acabam sendo idosos mais ativos no trabalho e tem tendência a serem mais longevos.

Também se identificou neste estudo um alto nível de sedentarismo nas idosas longevas dos grupos de convivência no domínio transporte. Cardoso, Mazo e Japiasú (2008) também encontraram valores semelhantes $(71,2 \%)$ de sedentarismo ao avaliarem 59 idosos com 60 anos e mais de idade. Os autores justificam esses achados às dificuldades de utilização de transporte, que se tornam maiores à medida que ocorre o envelhecimento, uma vez que nessa faixa etária coexistem uma série de morbidades e incapacidades.

Nas atividades domésticas as idosas longevas estudadas também foram consideradas em sua maioria sedentárias. Porém, quando comparados a outros estudos que utilizaram o mesmo instrumento, mas com idosos mais novos (60 anos e mais de idade), os resultados encontrados são divergentes, pois Cardoso, Mazo e Japiasú (2008) ao estudarem 59 idosos e Mazo et al. (2007) 256 idosos evidenciaram que eles são mais ativos nas atividades domésticas. Acredita-se que esses resultados são justificados pela faixa etária mais elevada de nossa amostra.

Os achados de nosso estudo corroboram com outras pesquisas que mostram que a maioria dos idosos não pratica nenhuma atividade física no lazer. No estudo denominado Epidoso, realizado com idosos residentes na Vila Clementino em São Paulo-SP, foi constatado que $73 \%$ dos 21 idosos eram considerados insuficientemente ativos no lazer, sendo que, entre eles, $57 \%$ não utilizavam nem dez minutos de seu tempo livre durante a semana em atividades físicas (NOVAIS, 2009). Valério (2011) fez o mesmo estudo na Vila Clementino em São Paulo-SP, e avaliou 494 idosos quanto ao nível de atividade física no domínio lazer, também utilizando o IPAQ, e verificou que $57 \%$ desses idosos não realizavam nem dez minutos de atividade física em seu tempo livre. Ambos os estudos foram realizados com idosos de 60 anos e mais. Em Florianópolis-SC, no estudo epidemiológico, com 875 idosos na faixa de idade entre 60 e 101 anos, de ambos os sexos, verificou que $74 \%$ desses idosos não realizavam nenhuma atividade física no tempo de lazer (BENEDETTI; PETROSKI; GONÇALVES, 2004). Essa característica tende a reforçar-se com o aumento da idade.

Essas altas prevalências de inatividade física por parte dos idosos é consequência da baixa porcentagem deles inseridos em programas de atividade física (BRASIL, 2009), 
sobretudo entre os idosos mais longevos. Assim, o grande desafio da promoção da saúde por meio da prática de atividade física para idosos é, além de romper com as barreiras para que eles possam começar a prática regular, fazer com que, uma vez iniciada, esses idosos mantenham o estilo de vida ativo (PRATT; EPPING; DIETZ, 2009).

\section{Conclusão}

A maioria das idosas longevas inativas fisicamente frequentadoras dos grupos de convivência de Florianópolis-SC era viúva, morava acompanhada, era católica, brancas, aposentada/pensionista. Apresentavam baixo nível de escolaridade, pois a maioria não concluiu o ensino fundamental. Com relação as condições de saúde apontaram ter pelo menos uma doença diagnosticada pelo médico, faziam uso de medicamentos regularmente e consideraram seu estado de saúde atual regular. Em relação ao nível de atividade física a maioria das idosas não praticava atividade física moderada ou vigorosa, por pelo menos 10 minutos contínuos durante a semana, nos quatro domínios do IPAQ.

Assim, espera-se que os resultados encontrados neste estudo possam contribuir para novas contextualizações/reflexões sobre as características sociodemográficas, as condições de saúde e o nível de atividade física das idosas longevas, onde esse conhecimento poderá auxiliar o planejamento de estratégias de intervenções que busquem modificações de comportamentos para a adoção de um estilo de vida ativo, onde a manutenção da atividade física pode resultar em uma série de benefícios físicos e sociais, o que proporcionará as idosas longevas, anos de vida com melhor capacidade funcional, condições de saúde e independência.

Compreender os mecanismos que tornam um idoso longevo e inativo fisicamente e o que pode ser feito para mudar esse comportamento é necessário e impreterível. Portanto, estratégias individuais e coletivas no âmbito da saúde pública são necessárias para modificar essa situação. Campanhas comunitárias, divulgações informativas, entrega de materiais e intervenções de apoio social em ambientes comunitários que expliquem sobre a importância e como realizar a atividade física, além da ampliação ao acesso aos locais de atividade física e de intervenções específicas com idosos longevos são ações que devem ser realizadas para que esta população mais envelhecida tenha um comportamento 
mais ativo e consequentemente usufruam dos benefícios biopsicossociais da atividade física. Sendo assim, novos estudos carecem de serem realizados envolvendo a população longeva, para que estes possam ter melhores condições de vida.

\section{Referências}

ACSM. AMERICAN COLLEGE OF SPORTS MEDICINE. Exercise and Physical Activity for Older Adults. Medicine Science of Sports and Exercise, Indianapolis (EUA), v. 41, n. 7, p. 1510-1530, 2009.

BENEDETTtI, T. R. B.; PETROSKI, E. L.; GONÇALVES, L. H. T. Perfil do idoso do município de Florianópolis: relatório final. Florianópolis: Pallotti, 2004. 88p.

BENEDETTI, T. R. B.; GONÇALVES, L. H. T.; MOTA, J. A. P. S. Uma Proposta de Política Pública de Atividade Física para Idosos. Texto e Contexto Enfermagem, Florianópolis, v. 16, n. 3, p. 387-398, jul./set., 2007.

BRASIL. Ministério da Saúde. Vigitel Brasil 2009: vigilância de fatores de risco e proteção para doenças crônicas por inquérito telefônico. Brasília: Ministério da Saúde, 2010.

CASSOU, A. C. N. et al. Barriers to Physical Activity Among Brazilian Elderly Women From Different Socioeconomic Status: A Focus-Group Study. Journal of Physical Activity and Health, Washington (EUA), v. 8, n. 1, p. 126 -132, jan., 2011.

CORSEUIL, M. W.; SCHNEIDER I. J. C.; SILVA, A. S.. et al. Perception of environmental obstacles to commuting physical activity in Brazilian elderly. Preventive Medicine, Amsterdã (HOL), v. 53, n. 4 e 5, p. 289-292, oct./Nov., 2011.

CARDOSO, A. S.; BORGES, L. J., MAZO, G. Z. et al. Fatores influentes na desistência de idosos em um programa de exercício físico. Movimento, Porto Alegre, v. 14, n. 01, p. 225-239, jan./fev., 2008.

CRUZ, I. B. M.; ALMEIDA, M. S. C.; SCHWANKE, C. H. A. et al. Prevalência de obesidade em idosos longevos e sua associação com fatores de risco e morbidades cardiovasculares. Revista da Associação Médica do Brasil, São Paulo, v. 50, n. 2, p.172-177, mar./abr., 2004. 
DUMITH, S. C. Proposta de um modelo teórico para a adoção da prática de atividade física. Revista Brasileira de Atividade Física e Saúde, Pelotas, v. 13, n. 2, p. 110-120, abr./jun., 2009.

GOBBI, S.; CARRITA, L. B.; HIRAYAMA, M. S. et al. Comportamento e Barreiras: Atividade Física em Idosos Institucionalizados. Psicologia: Teoria e Pesquisa, Brasília, v. 24, n. 4, p. 451-458, out./dez., 2008.

HALLAL, P. C.; VICTORA, C. G.; WELLS, J. C. K. et al. Physical Inactivity: Prevalence and Associated Variables in Brazilian Adults. Medicien \& Science in Sports \& Exercise, Indianapolis (EUA), v. 35, n. 11, p. 1894-1900, 2003.

HIRVENSALO, M.; LINTUNEN, T. Life-course perspective for physical activity and sports participation. European Review of Aging and Physical Activity, Munique (ALE), v. 8, p. 13-22, jan., 2011.

IBGE. INSTITUTO BRASILEIRO DE GEOGRAFIA E ESTATÍSTICA. Síntese de indicadores sociais - Uma análise das condições de vida da população brasileira. Rio de Janeiro: IBGE, 2010. Disponível em: http://www.ibge.gov.br/cidadesat/topwindow. htm?1> acesso em: 26 de junho de 2011.

JARDIM, S. E. G. Aspectos socioeconômicos do envelhecimento. In: PAPALÉO NETO, M. (Org.). Tratado de Gerontologia. São Paulo: Atheneu; 2007.

MARAFON, L. P.; CRUZ, I. B. M.; SCHWANKE, C. H. A. et al. Preditores cardiovasculares da mortalidade em idosos longevos. Cadernos de Saúde Pública, Rio de Janeiro, v. 19, n. 3, p. 799-808, jun., 2003.

MAZO, G. Z.; BENEDETTI, T. R. B. Adaptação do questionário internacional de atividade física para idosos. Revista Brasileira de Cineantropometria e Desempenho Humano, Florianópolis, v. 12, n. 6, p. 480-484, jun., 2010.

MAZO, G. Z.; LIPOSCKI, D. B.; ANANDA, C. et al. Condições de saúde, incidência de quedas e nível de Atividade física dos idosos. Revista Brasileira de Fisioterapia, São Carlos, v. 11, n. 6, p. 437-442, nov./dez, 2007.

MECHLING, H.; NETZ, Y. Aging and inactivity-capitalizing on the protective effect of planned physical activity in old age. European Revew of Aging and Physical Activity, Munique (ALE), v. 6, p. 89-97, jan., 2009. 
MEURER, S. T.; BENEDETTI, T. R. B.; MAZO, G. Z. Teoria da autodeterminação: compreensão dos fatores motivacionais e autoestima de idosos praticantes de exercícios físicos. Revista Brasileira de Atividade Física \& Saúde, Pelotas, v. 16, n. 1, p. 18-23, jan./ mar., 2011.

MOREIRA, L. M. A.; SOUZA, F. R.; CORDEIRO, A. P. B. et al. Estudo sobre a longevidade em Santa Inês, Bahia: Aspectos demográficos, genéticos e sociais. Revista de Ciências Médicas e Biológicas, Salvador, v. 6, n. 3, p. 331-337, set./dez., 2007.

MOSCHNY, A. et al. Barriers to physical activity in older adults in Germany: a crosssectional study. International Journal of Behavioral Nutrition and Physical Activity, Londres (ING), v. 8, dez., 2011.

NERI, A. L. Renda, consumo e aposentadoria: evidências, atitudes e percepções. In: NERI, A. L. (Org). Idoso no Brasil: vivências, desafios, e expectativas na terceira idade. São Paulo: Fundação Perseu Abramo, Edições SESC, 2007.

NOGUEIRA, S. L.; RIBEIRO, R. C. L.; ROSADO, L. E. F. P. L. et al. Fatores determinantes da capacidade funcional em idosos longevos. Revista Brasileira de Fisioterapia, São Carlos, v. 14, n. 4, p. 322-329, jun./jul., 2010.

NOVAIS, F. V. Nível de atividade física no lazer em idosos residentes na região da Vila Clementino no Município de São Paulo. 2009. 136. Dissertação (Mestrado em ciências), UNIFESP, São Paulo, 2009.

PATRICIO, K. P.; RIBEIRO, H.; HOSHINO, K. et al. O segredo da longevidade segundo as percepções dos próprios longevos. Revista Ciências e Saúde Coletiva, São Paulo, v. 13, n. 4, p. 1189-1198, abr., 2008.

PRATT, M.; EPPING, J. N.; DIETZ, W. H. Putting physical activity into public health: A historical perspective from the CDC, Preventive Medicine, Amsterdã (HOL), v. 49, p. 301302, jul., 2009.

PREFEITURAMUNICIPALDE FLORIANÓPOLIS. Perfil de Florianópolis. Florianópolis: PMF, 2009.

RIBEIRO, P. C. C.; NERI, A. L.; CUPERTINO, A. P. F. B. et al. Variabilidade no envelhecimento ativo segundo gênero, Idade e saúde. Psicologia em Estudo, Maringá, v. 14, n. 3, p. 501-509, jul./set., 2009. 
SCARMEAS, N.; LEVY, G.; TANG, M. X. et al. Influence of leisure activity on the incidence of Alzheimer's Disease. Neurology, Chicago (EUA), v. 57, n. 12, p. 2236-2242, dec., 2001.

SCHMIDT, J. A.; DAL-PIZZOL, F.; XAVIER, F. M. F. et al. Aplicação do teste do relógio em octogenários e nonagenários participantes de estudo realizado em Siderópolis/SC. Revista Psico, Porto Alegre, v. 40, n. 4, p. 525-530, out./dez., 2009.

SCHWANKE, C. H. A.; CRUZ,. I. B. M.; LEAL, N. F. et al. Análise da Associação entre Polimorfismo do Gene da Apolipoproteína E e Fatores de Risco Cardiovasculares em Idosos Longevos. Arquivos Brasileiros de Cardiologia, Rio de Janeiro, v. 78, n. 6, p. 561-570, dez., 2002.

SILVA, L. J. Avaliação do nível de atividade física, aptidão física e capacidade funcional de idosas participantes do programa de atividade física coordenado pelo programada de saúde da família do município de São Caetano do Sul. 2009. 132 f. Dissertação. (Mestrado em ciências), UNIFESP, São Paulo, 2009.

VALÉRIO, M. P. Estudo comparativo de intervenções voltadas à promoção de atividade física em idosos sedentários no lazer. 2011. 108 f. Tese (Doutorado em Ciências), UNIFESP, São Paulo, 2011.

VON BONSDORFF, M. B.; RANTANEN, T. Progression of functional limitations in relation to physical activity: a life course approach. European Review of Aging and Physical Activity, Munique (ALE), v. 8, p. 23-30, Aug., 2011.

XAVIER, F. M. F.; ARGIMON, I. I. L.; Zuppo, L. et al. O desempenho em testes neuropsicológicos de octagenários não-dementes e com baixa escolaridade em duas comunidades do sul do Brasil. Revista Psico, Porto Alegre, v. 37, n. 3, p. 221-231, set./ dez., 2006.

ZIBETTI, M. R.; GINDRI, G.; PAWLOWSKI, J. et al. Estudo comparativo de funções neuropsicológicas entre grupos etários de 21 a 90 anos. Revista Neuropsicologia Latinoamericana, Porto Alegre, v. 2, n. 1, p. 55-67, jan./abr., 2010. 


\section{SOCIODEMOGRAPHIC CHARACTERISTICS AND HEALTH CONDITIONS OF OLDEST OLD PHYSICALLY INACTIVE PARTICIPANTS OF LIVING GROUPS FOR THE OLDER ADULTS}

ABSTRACT: Aimed to identify the sociodemographic characteristics and health of oldest old physically inactive participants of living groups for the older adults of different regions of Florianópolis-SC. Were studied 173 older adults physically inactive, with aged 80 years or more attending of living groups of Florianópolis-SC. Was applied structured interview, containing sociodemographic date, health status and the International Physical Activity Questionnaire adapted for older adults. The mean age was $84.9 \pm 4.12$ years, being the majority widows, low education, catholic, caucasian, retired/pensioners, had some disease, used drugs and did not practiced moderate or vigorous physical activity for at least 10 continuous minutes during a normal week, in the four domains of the IPAQ. Expected that these results helping in planning intervention strategies that seek to modified of behavior for adopt an active lifestyle of this population.

Keywords: older adults aged over 80 years. Sociodemographic characteristics. Health status. Level of physical activity. 


\section{CARACTERÍSTICAS SOCIODEMOGRÁFICAS Y CONDICIONES DE SALUD DE MUJERES LONGEVAS FÍSICAMENTE INACTIVAS PARTICIPANTES DE GRUPOS DE CONVIVENCIA PARA PERSONAS MAYORES}

RESUMEN: El objetivo era identificar las características sociodemográficas y condiciones de salud de mujeres longevas físicamente inactivas participantes de grupos de convivencia para personas mayores de diferentes regiones de Florianópolis- SC. Fueron estudiados 173 mayores físicamente inactivos, con 80 años o más, que asisten a los grupos de convivencia de Florianópolis-SC. Se aplicó entrevista estructurada, que contiene características sociodemográficas, condiciones de salud y el International Physical Activity Questionnaire adaptados para las personas mayores. La edad media fue de 84,9 \pm 4,12 años, siendo la mayoría viuda, con bajo nivel de educación, católica, caucásico, jubiladas/pensionistas, tenían alguna enfermedad, utilizaban medicamentos y no practicaban actividad física moderada o vigorosa durante al menos 10 minutos continuos durante una semana normal, los cuatro dominios del IPAQ. Se espera que estos resultados ayuden en la planificación de estrategias de intervención que tratan de cambiar el comportamiento a adoptar un estilo de vida activo de esta población.

Palabras clave: Personas mayores de 80 años o más. Características sociodemográficas. Estado de la salud. Nivel de actividad física. 